\title{
Perfect Difference Networks and Related Interconnection Structures for Parallel and Distributed Systems
}

\author{
Behrooz Parhami, Fellow, IEEE, and Mikhail Rakov
}

\begin{abstract}
In view of their applicability to parallel and distributed computer systems, interconnection networks have been studied intensively by mathematicians, computer scientists, and computer designers. In this paper, we propose an asymptotically optimal method for connecting a set of nodes into a perfect difference network (PDN) with diameter 2, so that any node is reachable from any other node in one or two hops. The PDN interconnection scheme, which is based on the mathematical notion of perfect difference sets, is optimal in the sense that it can accommodate an asymptotically maximal number of nodes with smallest possible node degree under the constraint of the network diameter being 2. We present the network architecture in its basic and bipartite forms and show how the related multidimensional PDNs can be derived. We derive the exact average internode distance and tight upper and lower bounds for the bisection width of a PDN. We conclude that PDNs and their derivatives constitute worthy additions to the repertoire of network designers and may offer additional design points that can be exploited by current and emerging technologies, including wireless and optical interconnects. Performance, algorithmic, and robustness attributes of PDNs are analyzed in a companion paper.
\end{abstract}

Index Terms-Bipartite graph, bisection width, chordal ring, degree, diameter, hyperstar, interconnection network, low-diameter network, regular network, two-hop connectivity.

\section{INTRODUCTION}

great deal of research in parallel and distributed A processing has dealt with methods of interconnecting processors or nodes to achieve goals such as low-latency, high-bandwidth, and/or energy-efficient communication. Communication latency, bandwidth, or energy requirements depend not only on the network architecture, but also on a number of factors relating to applications and their data exchange characteristics. Hence, the challenge in interconnection network design is finding the right match between the communication needs of applications and technological capabilities and the limitations inherent in each architecture. This, in turn, explains the proliferation of implemented and proposed connectivity schemes, sometimes characterized as the sea of interconnection networks [19], [23].

At the application level, internode communication in a parallel or distributed system may occur via shared variables or message passing. In either case, a virtual complete connectivity is envisaged that allows any process / node to communicate with any other one. This connectivity pattern is modeled by a complete graph $K_{n}$ consisting of $n$ nodes, with every node linked to each of the other nodes by an edge. Thus, $K_{n}$ is a regular graph of node degree $n-1$ which is both node and edge-symmetric (i.e., nodes/edges are fully interchangeable via relabeling of nodes). Physically,

- B. Parhami is with the Department of Electrical and Computer Engineering, University of California Santa Barbara, Santa Barbara, CA 93106-9560. E-mail: parhami@ece.ucsb.edu.

- M. Rakov is with the Department of Computer Science, University of California Santa Barbara, Santa Barbara, CA 93106-5110.

E-mail:mikhrak@aol.com.

Manuscript received 1 Dec. 2003; revised 12 Aug. 2004; accepted 18 Oct. 2004; published online 22 June 2005.

For information on obtaining reprints of this article, please send e-mail to: tpds@computer.org, and reference IEEECS Log Number TPDS-0224-1203. complete-graph connectivity is difficult to provide, due to both the high cost of nodes with many communication channels and lack of scalability for system growth. However, a physical $K_{n}$ connectivity constitutes an ideal against which the communication performance of other architectures can be compared. This is because with $K_{n}$, no intermediate node or routing switch is involved in data exchanges, leading to direct conflict-free routing. The $n$-node network $K_{n}$ has $n(n-1) / 2$ links and a total communication bandwidth of $n(n-1) / 2$, assuming unitcapacity links.

At the other extreme from $K_{n}$, the simplest possible physical connectivity pattern is that of $n$-node ring $R_{n}$ (linear array or line graph, $L_{n}$, resulting from the removal of one link from $R_{n}$, is also of some interest). Here, each node has only two communication channels: two bidirectional ports for undirected ring or one input and one output port in the case of directed ring. The number of links is $n$, as is the aggregate network bandwidth with unit-capacity links. Data exchange is direct only between each node and one or two neighbor(s) and indirect in all other cases. An even-sized ring network is an example of bipartite graph, with even and odd-numbered nodes constituting the two parts and links always connecting nodes that are not in the same part. The complete graph is not bipartite, but $K_{n / 2, n / 2}$ can be defined (for even $n$ ) in which a node is connected to all nodes in the other part. This leads to 1 or 2-hop connectivity between each pair of nodes.

Intermediate architectures between $K_{n}$ and $R_{n}$ can be obtained in a variety of ways, providing tradeoffs in cost (affected by parameters such as node degree $d$ ) and performance (affected by network diameter $D$ and bisection bandwidth $B$, among other factors). Many of these intermediate architectures can be viewed as rings to which 


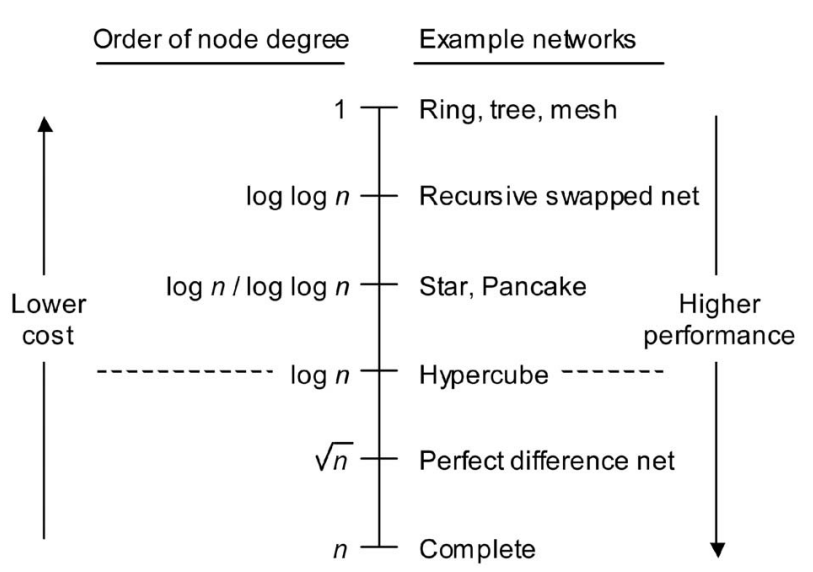

Fig. 1. The spectrum of networks in terms of node degree. The hypercube, with its excellent performance and logarithmic diameter, is often used as a reference point for comparisons.

bypass links or chords have been added in order to reduce the network diameter (e.g., chordal rings [3]) or richly connected graphs in which certain links are systematically removed through pruning [17], [24], [26] to reduce the network cost. Fig. 1 is an abstract view of the design space for interconnection networks (including perfect difference networks, introduced later in this paper) in terms of node degree varying from the minimum $\mathrm{O}(1)$ to the maximum $\mathrm{O}(n)$. Note that a node degree of $\mathrm{O}\left(n^{1 / 2}\right)$, while significantly better than $\mathrm{O}(n)$, still does not allow us to build large networks directly if wired connectivity is assumed. However, the situation is quite different with wireless and optical links. Furthermore, many large networks are built by combining smaller subnetworks or clusters in a manner to be discussed shortly. In such cases, where even the complete graph may be considered a viable cluster connectivity scheme for relatively small clusters, perfect difference networks allow us to move to somewhat larger clusters with negligible increase in internode distances.

Three general mechanisms for obtaining a variety of useful networks from smaller component networks are the cross product, recursive substitution, and hierarchical composition of graphs.

The cross product (Cartesian product or simply product) of the $q$ graphs, $G_{i}=\left(V_{i}, E_{i}\right), 0 \leq i \leq q-1$, denoted as $G_{q-1} \times G_{q-2}$ $\times \ldots \times G_{0}$, is a graph with $n_{q-1} \times n_{q-2} \times \ldots \times n_{0}$ nodes, each labeled with a distinct $q$-digit mixed-radix integer $x_{q-1} x_{q-2}$ $\ldots x_{0}$ in the range 0 to $n_{q-1} \times n_{q-2} \times \ldots \times n_{0}-1$, so that nodes $x$ and $y$ are connected iff their labels differ only in one digit, say $x_{j} \neq y_{j}$, and $x_{j}$ is connected to $y_{j}$ in $G_{j}$ [45]. The node degree of a product graph is the sum $d_{q-1}+d_{q-2}+\ldots+d_{0}$ of the node degrees for the component graphs and its diameter is the sum $D_{q-1}+D_{q-2}+\ldots+D_{0}$ of the diameters. A wide variety of networks can be built through cross-product composition. For instance, the $q$-cube ( $q$-dimensional binary hypercube) is $K_{2} \times K_{2} \times \ldots \times K_{2}$. As a further example, the product of two $n^{1 / 2}$-node complete graphs is a network of node degree $2 n^{1 / 2}-2$ and diameter 2 . More generally, $K_{m} \times$ $K_{m} \times \ldots \times K_{m}$ is referred to as $q$-dimensional radix- $m$ generalized hypercube. It has $n=m^{q}$ nodes, with node degree $d=$ $q(m-1)$ and diameter $D=q$.

Multilevel hierarchical networks come in several different flavors [25]. In all of these, $h$ different or identical graphs
$G_{q-1}, G_{q-2}, \ldots, G_{0}$ are involved, which define the connectivity patterns at the $h$ levels of the hierarchy. In the topdown recursive substitution scheme, we start with $G_{q-1}$ and replace each of its nodes with a graph $G_{q-2}$, forming a supernode. Within each supernode, we use an agreed upon scheme to connect the edges of $G_{q-1}$ to the nodes of $G_{q-2}$. The process can be repeated with the resulting graph, replacing each node in the composition of $G_{q-1}$ and $G_{q-2}$ with $G_{q-3}$ and so on. In the bottom-up hierarchical composition scheme, we start with several copies of the nucleus graph $G_{0}$, which form supernodes for the next-level graph $G_{1}$. The composition of $G_{0}$ and $G_{1}$ then defines the structure of supernodes within $G_{2}$, and so on.

One of the main foci in research on interconnection networks over the past two decades has been the exploration of the design space depicted in Fig. 1, with particular emphasis on deriving networks with sublogarithmic degrees that can provide some of the desirable properties of the hypercube [1], [9], [18], [29], [44]. Emphasis on sublogarithmic-degree networks was justified by VLSI area requirements and packaging constraints, including pin limitations; i.e., cost and realizability factors. Perfect difference networks, which form the primary focus of this paper, provide us with design points on the other side of the hypercube in Fig. 1. They offer the benefits of full connectivity at a much lower cost. The part of design space that falls between the hypercube and $K_{n}$ is of little interest in architectures with wired connectivity [6], [46], but becomes more practical and, thus, interesting with wireless or optical links.

\section{Evaluation Criteria for Networks}

Network diameter $D$, defined as the longest of the internode distances, is an important figure of merit for networks. The diameter $D$ indicates the worst-case number of hops in sending a message from one node to another. The diameter of a network ranges from the best of 1 for $K_{n}$ to the worst of $n-1$ for the $n$-node linear array or line-graph $L_{n}$. That the worst-case latency for messages in a network is highly dependent on $D$ is obvious with store-and-forward routing. It is somewhat less clear that, even with wormhole switching [10], [21], [22], network diameter plays a key role in communication latency, albeit in an indirect way. This is best understood by considering the case of short and long messages separately. For short worms, the travel time of the head, which is proportional to the hop distance, dominates the overall message latency. For long messages, a significant number of links, perhaps the entire source-to-destination path, is occupied by the worm carrying the message. In networks with large diameters, the worms tend to be longer and, thus, occupy a greater portion of the aggregate network bandwidth. This either increases the possibility of deadlock or else forces us to use less aggressive routing algorithms. Either alternative implies lower performance. For a more detailed exposition of the importance of network diameter, see [28].

Average internode distance $\Delta$ is defined as the average of the lengths of the distances between all $n(n-1)$ pairs of nodes, or perhaps between all $n^{2}$ pairs of nodes when the distance of each node to itself is also included in the 
averaging. The average internode distance $\Delta$ is representative of average or expected communication latencies, whereas $D$ represents the worst case. However, for virtually all interconnection networks of practical interest, $D$ and $\Delta$ are very closely related, so that they are practically interchangeable for use as a figure of merit. For example, in all node-symmetric networks and a wide variety of nodeasymmetric networks, we have $D / 2 \leq \Delta \leq D$ [28]. For such networks, the average internode distance $\Delta$ generally grows in proportion to $D$, even though the relationship between the two parameters is not strictly linear. Put another way, if the diameter of the network is quadrupled, the average internode distance at least doubles.

The bisection width $B$ of a network is the minimum number of links whose removal cuts the network in two parts, with $\lfloor n / 2\rfloor$ nodes on one side of the partition and $\lceil n / 2\rceil$ nodes on the other. Bisection bandwidth is defined in terms of link bandwidths, rather than multiplicity, for networks in which the links have varying communication capacities. A large bisection (band)width is an indicator of large aggregate network capacity for routing random traffic patterns between arbitrary network nodes. For example, the hypercube with a bisection width of $n / 2$ can achieve a higher communication performance for random traffic than a 2D mesh with bisection width $n^{1 / 2}$. The mesh is, in turn, better than constant-bisection networks such as rings and trees.

It is, of course, not very practical to consider $D, \Delta$, or $B$ in isolation. The complete graph $K_{n}$ is theoretically ideal because it has the best possible parameters $D=\Delta=1$ and $B=n(n-1) / 2$. So, we must view network parameters in the context of network cost. Because real network cost is very difficult to predict and model, abstract notions of cost have been proposed to allow more practical network comparison methodologies. These abstract notions vary in complexity and, thus, accuracy. Some simple cost factors include node degree $d$, total number of links (which is $n d / 2$ for a regular degree- $d$ network), and the square of bisection width (because the VLSI layout area is lower-bounded by $B^{2}$ ). More complex cost factors take the modularity of the network, which has a bearing on partitioning and packaging costs, into account as well.

Composite figures of merit, that take both topological parameters and one or more cost factors into account, have also been proposed. For example, the product $d D$ (degreediameter product) has been widely used for comparing networks. This measure favors networks that achieve small diameters with low cost (node degree). According to this measure, $K_{n}$, having degree-diameter product of $n-1$, is inferior to a $2 \mathrm{D}$ square torus with $d D=4 n^{1 / 2}$. The hypercube with $d D=\log ^{2} n$ is asymptotically better than both $K_{n}$ and 2D square torus.

Based on the foregoing discussion, it is clearly desirable to build networks of the smallest possible diameters with a given node degree $d$. For decades, graph theorists have studied the problem of synthesizing minimum-diameter graphs with a given maximum node degree. The problem is still open, but a number of bounds, and graphs that come close to the optimal bounds, are known.

Consider an $n$-node directed graph (digraph) with nodes of constant in-degree and out-degree $d$. The number of different nodes that can be reached from a given node in $D$

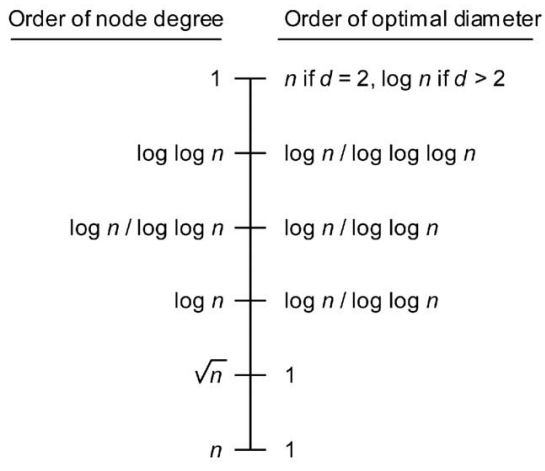

Fig. 2. Optimal diameter in terms of node degree (orders of magnitude shown without constants of proportionality).

or fewer steps is at most $1+d+d^{2}+\ldots+d^{D}$. This establishes an upper bound on the number of nodes for a given diameter $D$ or a lower bound on the diameter for a given number $n$ of nodes:

$$
\begin{aligned}
& n \leq\left(d^{D+1}-1\right) /(d-1) \\
& D \geq \log _{d}[n(d-1)+1]-1 .
\end{aligned}
$$

These inequalities are known as Moore bounds. Any digraph matching these bounds is a Moore digraph. Moore digraphs are of interest because they exhibit the lowest possible degree-diameter product for a given node degree $d$ or diameter $D$, when the number $n$ of nodes is fixed. Corresponding bounds for undirected graphs can also be obtained [23]. According to Moore's bound, an $n$-node degree- $d$ digraph must have a diameter that grows at least as $\log _{d} n$. The variation of the optimal diameter with node degree is depicted in Fig. 2 in terms of orders of magnitude. We note that logarithmic diameter can be achieved with constant node degree. As the node degree increases, the diameter is reduced, but not substantially. When the node degree has grown to $\mathrm{O}(\log n)$, the diameter is only divided by $\log \log n$. To have a constant diameter, the node degree must become $\mathrm{O}\left(n^{\varepsilon}\right)$, with the constant diameter being larger for smaller values of $\varepsilon$.

\section{Perfect Difference Sets}

Given that the complete graph $K_{n}$ (with diameter $D=1$ ) is impractical for large $n$, it is quite natural to consider the best topology for $D=2$, the next most desirable network diameter. Based on Moore bounds, a degree- $d$ digraph with $D=2$ can have no more than $n=d^{2}+d+1$ nodes. The corresponding upper bound $n=d^{2}+1$ for undirected graphs is not much different [23]. Examples of diameter-2 networks of small sizes include the Petersen and HoffmanSingleton networks [7]. Perfect difference sets provide the mathematical tools for achieving this optimum number of nodes, in an asymptotic manner, within the framework perfect difference networks or PDNs (see Fig. 1 for the place of PDN in the spectrum of network choices).

It should be noted that the name "hyperstar" [30], [31], [34] was originally coined for what we call "PDN" in this paper. The change of name to the more descriptive PDN was triggered by our desire to avoid confusion with networks [2] and various commercial hardware and soft- 
TABLE 1

Perfect Difference Sets of Orders Up to 16

\begin{tabular}{|c|c|c|}
\hline $\boldsymbol{\delta}$ & $\boldsymbol{n}$ & Example PDS of order $\boldsymbol{\delta}$ in normal form \\
\hline 2 & 7 & $0,1,3$ \\
\hline 3 & 13 & $0,1,3,9$ \\
\hline 4 & 21 & $0,1,4,14,16$ \\
\hline 5 & 31 & $0,1,3,8,12,18$ \\
\hline 7 & 57 & $0,1,3,13,32,36,43,52$ \\
\hline 8 & 73 & $0,1,3,7,15,31,36,54,63$ \\
\hline 9 & 91 & $0,1,3,9,27,49,56,61,77,81$ \\
\hline 11 & 133 & $0,1,3,12,20,34,38,81,88,94,104,109$ \\
\hline 13 & 183 & $0,1,3,16,23,28,42,76,82,86,119,137,154,175$ \\
\hline 16 & 273 & $0,1,3,7,15,31,63,90,116,127,136,181,194,204,233,238,255$ \\
\hline
\end{tabular}

Note that the values of $\delta$ shown are powers of prime numbers and $n=\delta^{2}+\delta+1$.

ware products that already use the name "hyperstar." A class of multidimensional PDNs (introduced in Section 5), which was previously referred to as "hyperhub" [32], [33], was renamed for the same reasons.

Perfect difference sets were first discussed by Singer in 1938 [38]. The formulation was in terms of points and lines in a finite projective plane. The theory of finite projective planes is highly developed [15], but these mathematical notions are not required to understand the exposition that follows. We first present a theorem that forms the basis of the definition of perfect difference sets and, then, the definition itself. All results in this section are from [38]. Additional information on difference sets can be found in [14], [40], [43].

Theorem 1. A sufficient condition that there exist $\delta+1$ integers $s_{0}, s_{1}, \ldots, s_{\delta}$, having the property that their $\delta^{2}+\delta$ differences $s_{i}-s_{j}, i \neq j, 0 \leq i, j \leq \delta$, are congruent, modulo $\delta^{2}+\delta+1$, to the integers $1,2, \ldots, \delta^{2}+\delta$ in some order is that $\delta$ be a power of a prime.

Definition 1. Perfect difference set (PDS) - A set $\left\{s_{0}, s_{1}, \ldots, s_{\delta}\right\}$ of $\delta+1$ integers having the property that their $\delta^{2}+\delta$ differences $s_{i}-s_{j}, 0 \leq i \neq j \leq \delta$, are congruent, modulo $\delta^{2}+\delta+1$, to the integers $1,2, \ldots, \delta^{2}+\delta$ in some order is a perfect difference set of order $\delta$. Perfect difference sets are sometimes called simple difference sets, given that they correspond to the special $\lambda=1$ case of difference sets for which each of the possible differences is formed in exactly $\lambda$ ways.

Note that a PDS need not contain an integer outside the interval $\left[0, \delta^{2}+\delta\right]$ because any integer outside the interval can be replaced by another integer in the interval without affecting the defining property of the PDS. The following is easily proven.

Theorem 2. Given a PDS $\left\{s_{0}, s_{1}, \ldots, s_{\delta}\right\}$ of order $\delta$, the set $\left\{a s_{0}+b, a s_{1}+b, \ldots, a s_{\delta}+b\right\}$, where $a$ is relatively prime to $\delta^{2}+\delta+1$, also forms a perfect difference set.

By definition, any perfect difference set contains a pair of integers $s_{u}$ and $s_{v}$ such that $s_{v}-s_{u} \equiv 1 \bmod \delta^{2}+\delta+1$. By Theorem 2 and the observation that preceded it, subtracting $s_{u}$ from all integers in such a PDS yields another PDS that contains 0 and 1 .
Definition 2. Normal PDS-A PDS $\left\{s_{0}, s_{1}, \ldots, s_{\delta}\right\}$ is reduced if it contains the integers 0 and 1 . A reduced PDS is in normal form if it satisfies $s_{i}<s_{i+1} \leq \delta^{2}+\delta, 0 \leq i<\delta$.

Definition 3. Equivalent PDSs-Two different PDSs are equivalent iff they have the same normal form $\left\{0,1, s_{2}, \ldots, s_{\delta}\right\}$.

Henceforth, we deal only with PDSs in normal form, some examples of which appear in Table 1.

Several properties of PDSs are worth noting.

Property 1. Existence-Theorem 1 guarantees that a PDS exists for any number $n$ that is of the form $\delta^{2}+\delta+1$, where $\delta=p^{h}$ and $p$ is a prime number. It is suspected, though not yet proven for arbitrarily large values of $n$, that PDSs do not exist for other values of $n$ [11], [13]. However, practically speaking, this is not alarming, given that primes and their powers are quite abundant, both in the range of practical interest for interconnection network size and asymptotically. For example, there are 197 primes and powers of primes under 1,000.

Property 2. Multiplicity-For some values of $\delta$, there exist more than one PDS. For example, we have the following PDSs of order $\delta=3$ :

$$
0,1,3,9 \text { and } 0,1,4,6 \text {. }
$$

It is easily verified that all numbers in the interval [1, 12] can be formed as the mod-13 difference of numbers in each of the two sets above:

$$
\begin{aligned}
& 1 \equiv 1-0 \equiv 1-0 \\
& 2 \equiv 3-1 \equiv 6-4 \\
& 3 \equiv 3-0 \equiv 4-1 \\
& 4 \equiv 0-9 \equiv 4-0 \\
& 5 \equiv 1-9 \equiv 6-1 \\
& 6 \equiv 9-3 \equiv 6-0 \\
& 7 \equiv 3-9 \equiv 0-6 \\
& 8 \equiv 9-1 \equiv 1-6 \\
& 9 \equiv 9-0 \equiv 0-4 \\
& 10 \equiv 0-3 \equiv 1-4 \\
& 11 \equiv 1-3 \equiv 4-6 \\
& 12 \equiv 0-1 \equiv 0-1
\end{aligned}
$$

We will see later that multiple difference sets of the same order lead to alternate interconnection network designs.

Property 3. Generation-A PDS of order $\delta=p^{z}$, where $p$ is a prime number, represents a set of $n$ points and $n$ lines in the 
$3 D$ Euclidian space such that each point is on $\delta+1$ lines and each line contains $\delta+1$ points. This geometric interpretation leads to a PDS of order $\delta=p^{z}$ being generated from an irreducible degree-3 polynomial in $\operatorname{GF}\left(p^{z}\right)$. Details are beyond the scope of this paper [38]. Here, we take it for granted that a $P D S$ of order $\delta=p^{z}$ can be easily generated when required.

Property 4. Relationship with perfect partitions-PDSs are closely related to perfect partitions, which have an even longer history [16]. Take any PDS in normal form and find the mod- $n$ differences $s_{i+1}-s_{i}$ between consecutive numbers in it, including the difference $s_{0}-s_{\delta}$. For example:

$$
\begin{array}{ll}
\operatorname{PDS}(\delta=3, n=13): & 0,1,3,9 \\
\text { mod- } n \text { differences } s_{i+1}-s_{i}: & 1,2,6,4 .
\end{array}
$$

Viewing this last sequence of integers as a circular one and adding subsequences of length 1, 2, and 3 beginning with each term, yields each of the sums in the interval [1,12] exactly once.

$$
\begin{aligned}
1 & =1 \\
2 & =2 \\
3 & =1+2 \\
4 & =4 \\
5 & =4+1 \\
6 & =6 \\
7 & =4+1+2 \\
8 & =2+6 \\
9 & =1+2+6 \\
10 & =6+4 \\
11 & =6+4+1 \\
12 & =2+6+4
\end{aligned}
$$

Such a mod-n sequence, which is also known as a perfect partition, ideal code, or ideal ring proportions, can be used in synthesizing PDN-type structures [35], [36]. However, PDSs provide a more straightforward and efficient tool in this regard. Note that a PDS is transformed to a corresponding perfect partition via modular subtraction of consecutive terms, while the reverse transformation involves computing modular prefix sums.

We conclude this section by referring to some applications. A PDS allows us to express a large set of integers via a set of much smaller size, in a simple and highly regular fashion. When used in the design of interconnection networks, this property translates to reduced number of links and switching elements or to more efficient use of bandwidth. In free-space optical communication, where physical links do not exist, use of PDS alleviates the precision requirements on positioning and reflecting elements or, alternatively, accommodates more channel with the prevailing physical tolerances. Aside from enabling useful interconnection structures and networks, as discussed here and in Section 4, perfect difference sets can be applied to a variety of other design problems. Examples include highly efficient error control codes [20], [37], block designs, which are related to orthogonal Latin squares and find applications in scheduling and design of experiments [4], and signal encoding to ensure negligible autocorrelation and cross-correlation for ease of decoding and separation [39], [41], [42]. These applications may be characterized by their need for provision of distance, variety, and/or

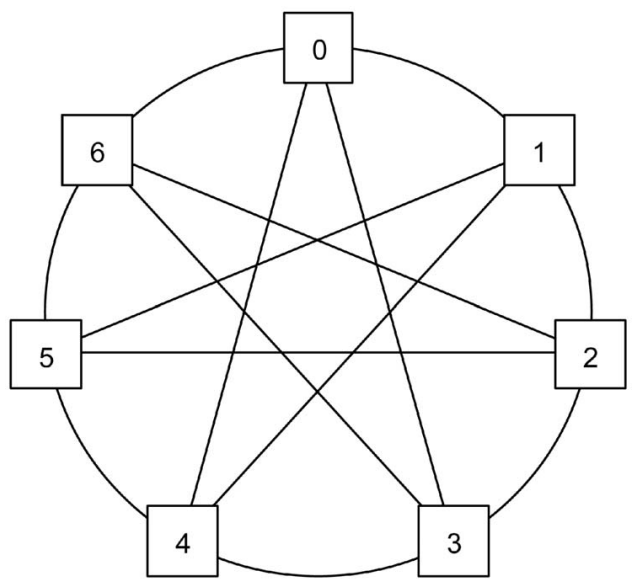

Fig. 3. The chordal ring structure of the PDN with $n=7$ nodes based on the perfect difference set $\{0,1,3\}$.

orthogonality, or for avoiding coincidence, all of which are facilitated by unique differences in a PDS.

\section{Perfect Difference Networks}

Consider the normal-form PDS $\left\{0,1, s_{2}, \ldots, s_{\delta}\right\}$ of order $\delta$. We can construct a direct interconnection network with $n=$ $\delta^{2}+\delta+1$ nodes based on this PDS as follows:

Definition 4. Perfect difference network (PDN) based on the PDS $\left\{0,1, s_{2}, \ldots, s_{\delta}\right\}$-There are $n=\delta^{2}+\delta+1$ nodes, numbered 0 to $n-1$. Node $i$ is connected via directed links to nodes $i \pm 1$ and $i \pm s_{j}(\bmod n)$, for $2 \leq j \leq \delta$. Given that all index expressions in this paper are evaluated modulo $n$, henceforth, we will delete the qualifier "mod $n "$ in our presentation. The preceding connectivity leads to a chordal ring of in and out-degree $d=2 \delta$ and diameter $D=2$ (this is justified later). Because, for each link from node $i$ to node $j$, the reverse link from node $j$ to node $i$ also exists, the network can be drawn as an undirected graph.

An example PDN for $n=7$, based on the PDS $\{0,1,3\}$, is depicted in Fig. 3.

Every normal-form PDS contains 1 as a member. Therefore, PDNs based on normal-form PDSs are special types of chordal rings. In the terminology of chordal rings, the links connecting consecutive nodes $i$ and $i+1$ are ring links, while those that connect nonconsecutive nodes $i$ and $i+s_{j}, 2 \leq j \leq \delta$, are skip links or chords. The link connecting nodes $i$ and $i+s_{j}$ is a forward skip link of node $i$ and a backward skip link of node $i+s_{j}$. Similarly, the ring link connecting nodes $i$ and $i+1$ is a forward ring link for $i$ and backward ring link for $i+1$.

As seen in Fig. 3, any two nodes in a PDN are either connected by a link directly or via a path of length 2 through an intermediate node. This property is elaborated upon in Fig. 4, where a shortest path from node 0 to each of the other nodes is highlighted and labeled with the associated difference $s_{i}-s_{j}$. Given the node symmetry of the network, shortest paths between other pairs of nodes are obtained by simply adding the index of the source node to all path labels seen in Fig. 4. As usual, the addition is modulo $n$. 


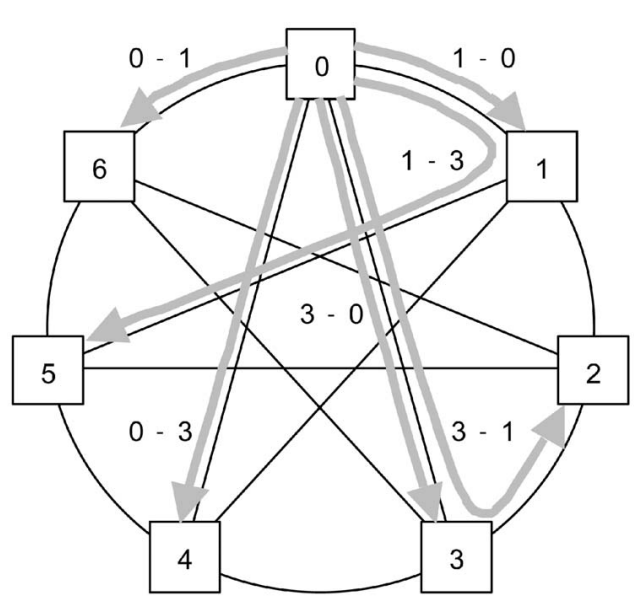

Fig. 4. The perfect difference network of Fig. 3, with shortest paths from node 0 to all others highlighted and labeled with corresponding differences.

There is an alternate way in which we can formulate an interconnection structure based on the normal-form PDS $\left\{0,1, s_{2}, \ldots, s_{\delta}\right\}$ of order $\delta$ [33]. This alternate scheme was briefly discussed in [5], but the filing of the patent in [30] and the second author's prior work that led to the patent predate [5]. The second author's prior work in this area, and the ensuing patents, are based on the following.

Definition 5. Bipartite PDN based on the PDS $\left\{0,1, s_{2}, \ldots\right.$, $\left.s_{\delta}\right\}$-Thereare $n=\delta^{2}+\delta+1$ host nodes, numbered 0 to $n-1$, and similarly numbered switch nodes. Each host node $i$ is connected via a pair of directed links to each of the switch nodes $i$, $i+1$, and $i+s_{j}$, for $2 \leq j \leq \delta$. The preceding connectivity leads to a bipartite network, with host and switch nodes constituting the two parts. Both nodes and switches have in and out-degrees $\delta+1$. The host-to-host network diameter is $D=2$. All host-to-host shortest paths are of length 2, leading to the average interhost distance $\Delta=2$. Again, the bipartite network can be drawn as an undirected graph.

An example bipartite PDN for $n=7$, based on the PDS $\{0,1,3\}$, is depicted in Fig. 5. An alternate drawing, with shortest paths from host node 0 highlighted, is shown in

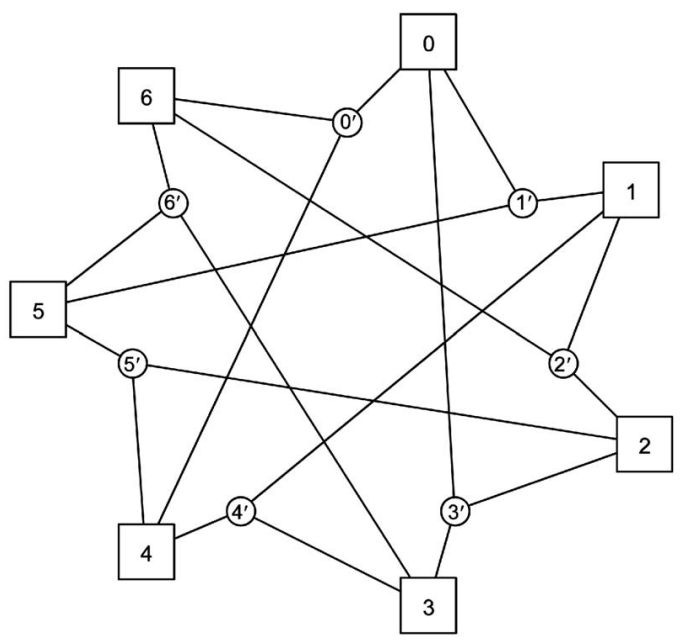

Fig. 5. Bipartite version of PDN with seven hosts (squares) and seven switches (circles), based on the perfect difference set $\{0,1,3\}$.
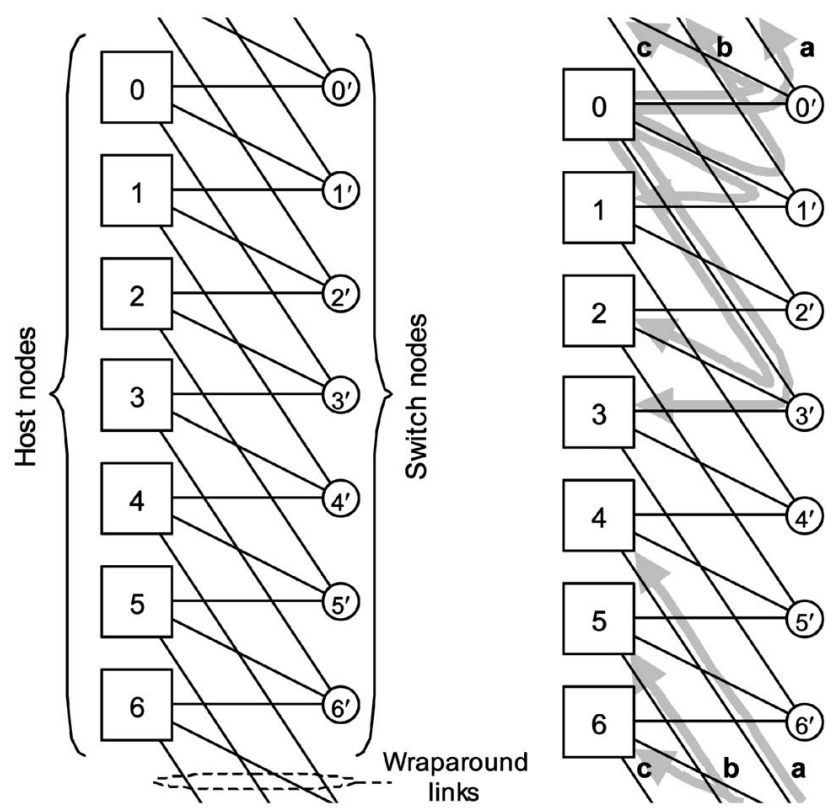

Fig. 6. Alternate view of bipartite PDN of Fig. 5, with paths from node 0 to all other nodes highlighted on the right.

Fig. 6. One advantage of a bipartite PDN over a basic PDN is that its node degree is reduced from $2 \delta$ to $\delta+1$ through the use of $n$ switches, with each switch being a $(\delta+1) \times$ $(\delta+1)$ communication node with full-crossbar or partial connection capability. The bipartite PDN can be viewed as simply a method for implementing the basic PDN. This is easily understood by drawing boxes around similarly numbered host and switch nodes in Fig. 5 and considering each such pair a node of degree $2 \delta$ within a basic PDN.

It is also possible to interpret the bipartite PDN as a $2 n$-node, degree- $(\delta+1)$ network by simply viewing all nodes in Fig. 5 as host nodes. The resulting network has a diameter of 3. This is easily seen as follows: The host nodes replacing the original switch nodes are denoted by primed indices. Each such primed node is directly connected to several unprimed nodes and any pair of unprimed nodes are connected by a shortest path of length no greater than 2 . For example, node 0 in Fig. 5 is not connected to node $2^{\prime}$ by any path of length shorter than 3 , but there are several paths of the latter kind: $01^{\prime} 12^{\prime}, 03^{\prime} 22^{\prime}, 00^{\prime} 62^{\prime}$. These paths are node- and edge-disjoint. In general, there would exist $\delta+1$ such paths through all switches connected to the source node, given that the interswitch diameter is also 2 .

\section{Multidimensional PDNs}

The perfect difference network, with its $\mathrm{O}\left(n^{1 / 2}\right)$ node degree in both its basic and bipartite forms, falls between the hypercube and complete graph in the design space of Fig. 1, offering performance close to the latter, at a much lower cost. If further cost reduction is desired, networks of smaller node degrees can be built based on the PDN concept. These networks fall in the space between hypercube and PDN in Fig. 1, offering somewhat lower performance than the latter at reduced cost, thus allowing cost-performance tradeoffs in numerous configurations. 


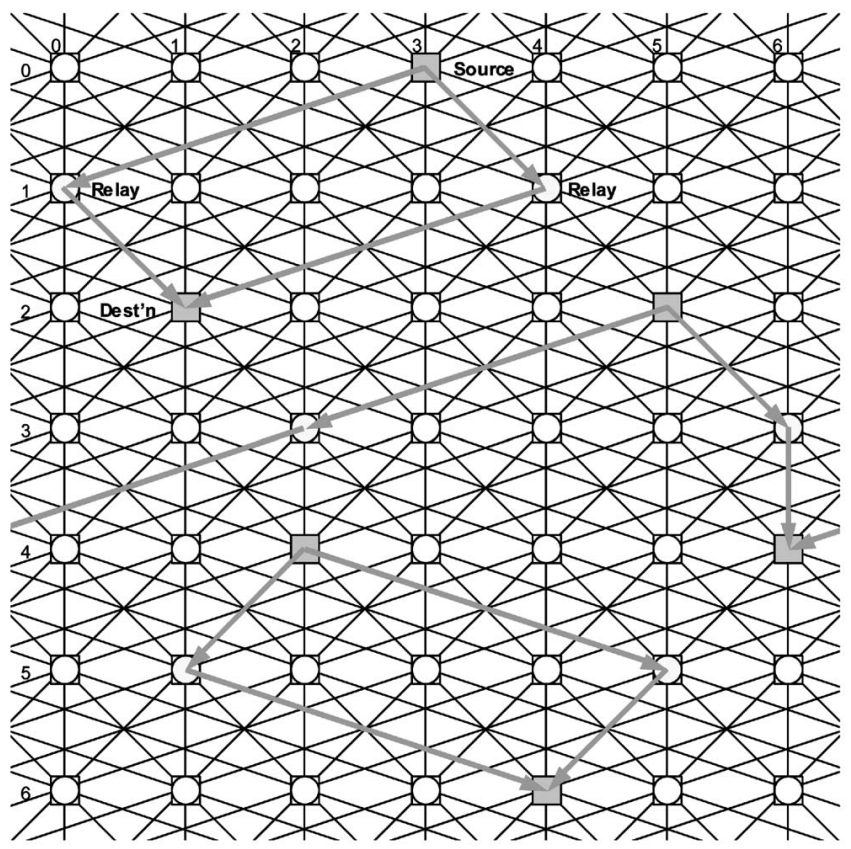

Fig. 7. Two-dimensional PDN fabrics, emphasizing the dual roles of nodes for initiating and facilitating internode communications.

A key mechanism for such trade offs is the multidimensional PDN, defined later in this section. However, before introducing this class of networks, it is instructive, both for further understanding of basic PDNs of Section 4 and for visualizing their properties with regard to computation and communication, to introduce a 2D representation which we refer to as PDN fabrics. Fig. 7 is the 2D fabrics corresponding to the 7-node PDN of Fig. 3.

Definition 6. PDN fabrics-Consider an n-node PDN H. Draw the $n$ nodes of $H$ in a horizontal arrangement and replicate the row arrangement $r$ times in the vertical direction. Instead of connecting the row nodes to each other according to the connectivity rules of $H$, connect each node to its counterparts and its "neighbors" in the preceding and succeeding rows, wrapping around as needed (see Fig. 7). Just as the number $r$ of rows is arbitrary, the columns too can be replicated to the right and to the left, as desired, by simply repeating the same pattern and node numbering, with the wraparound links becoming regular ones.

It is easily seen that the node degree in the PDN fabrics is $2 d+2=4 \delta+2$, where the original PDN $H$ has node degree $d=2 \delta$ and $n=\delta^{2}+\delta+1$ nodes. Note that the PDN fabrics of Definition 6 is a bipartite network, with even and oddnumbered rows constituting the two parts. PDN fabrics can be considered as corresponding to an $n r$-node network ( $n$ columns, $r$ rows). It can also be viewed as an unfolding of a simple PDN interconnection pattern that helps us see different internode paths which now become paths between nodes in different rows. This is helpful because it can be used for visualization of the time dimension in space.

Definition 7. PDN fabrics network-An $r$-row, $n$-column segment of the 2D PDN fabrics (as in Fig. 7), with wraparound connections between nodes at the two extremes of the same row or column is a PDN fabrics network.

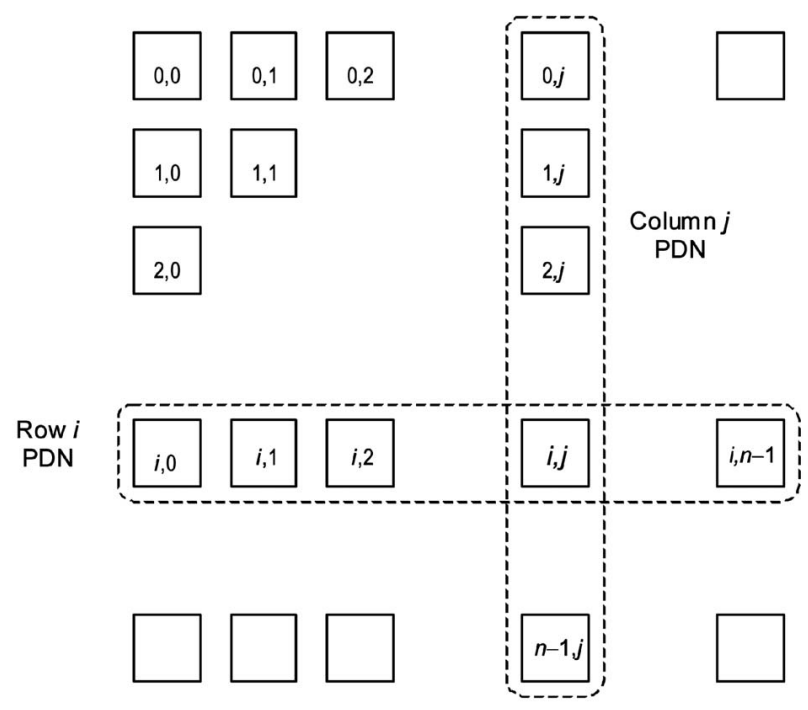

Fig. 8. The structure of the 2D PDN power network $H^{2}$.

Definition 8. Multidimensional PDN-Consider the $q$ PDNs $H_{0}, H_{1}, \ldots, H_{q-1}$ based on their respective PDSs of orders $\delta_{0}, \delta_{1}, \ldots, \delta_{q-1}$. The product network $H_{q-1} \times H_{q-2} \times \ldots \times H_{0}$ is a $q D$, or q-dimensional, PDN. Nodes of a $q D$ PDN are labeled by q-tuples $\left(x_{q-1} x_{q-2} \ldots x_{0}\right)$, where $x_{i}$ belongs to the node set of $H_{i}, 0 \leq i<q$. When the $q$ component PDNs $H_{i}$ are identical, the resulting network $H^{q}$ is a PDN-based power network.

For concreteness, we limit our subsequent discussion to 2D PDN-based power network $H^{2}$, depicted in Fig. 8. The statements that follow are easily generalizable to higher dimensions and nonidentical component PDNs.

To avoid clutter, the node interconnections within rows and columns are not shown in Fig. 8. Nodes in each row $i$ (column $j$ ) are linked together exactly at those in an $n$-node PDN. Therefore, the total number of links in Fig. 8 is a factor of $2 n$ greater than the number of links in the $n$-node PDN used as its basis. Hence, we can say that increasing the number of nodes by a factor of $n$ using multidimensional PDN has led to a factor $\mathrm{O}(n)$ increase in the total number of links. By contrast, had we opted for an $\mathrm{O}\left(n^{2}\right)$-node $\mathrm{PDN}$, its $\mathrm{O}\left(n^{3}\right)$ links would have been a factor of $\mathrm{O}\left(n^{3 / 2}\right)$ higher that the corresponding number for an $n$-node PDN.

Based on the properties of product graphs, the diameter of $H^{2}$ is 4 and its node degree is $4 \delta$, where $\delta$ is the order of the PDS defining $H$. If each row/column PDN has $n$ nodes, the 2D PDN power network will have $N=n^{2}$ nodes of degree $\mathrm{O}\left(n^{1 / 2}\right)$. Thus, node degree of $H^{2}$ grows as the fourth root of its size $N$. For example, a PDN with roughly $10^{6}$ nodes requires node degree of about 2,000, whereas a 2D PDN power network of the same size can be built of nodes with degrees that are about 16 times smaller. An immediate consequence of this slower growth of node degree is that $H^{2}$ has a much more favorable degree-diameter product than a simple PDN of comparable size (see Table 2). Asymptotically, the $d D$ factors are $16 \delta$ for $H^{2}$ versus $4\left(\delta^{2}+\delta+1\right)$ for the equivalent $H^{\prime}$, with the former being better except for $\delta=2$.

Because $H^{2}$ is a power network, all algorithmic properties of power networks are applicable to it. For example, routing 
TABLE 2

Degree-Diameter Product for $H^{2}$ Compared to a PDN $H^{\prime}$ of Comparable Size (Closest Possible Size to $n^{2}$ Nodes)

\begin{tabular}{|c|c|c|c|c|c|c|}
$\boldsymbol{\delta}$ & $\boldsymbol{n}$ & $\boldsymbol{H}^{\mathbf{2}}$ degree & $\boldsymbol{H}^{\mathbf{2}} \mathbf{d D}$ & $\boldsymbol{H}^{\prime}$ nodes & $\boldsymbol{H}^{\prime}$ degree & $\boldsymbol{H}^{\prime} \mathbf{d D}$ \\
\hline 2 & 7 & 8 & 32 & 57 & 14 & 28 \\
\hline 3 & 13 & 12 & 48 & 183 & 26 & 52 \\
\hline 4 & 21 & 16 & 64 & 553 & 46 & 92 \\
\hline 5 & 31 & 20 & 80 & 993 & 62 & 124 \\
\hline 7 & 57 & 28 & 112 & 3541 & 118 & 236 \\
\hline 8 & 73 & 32 & 128 & 5403 & 146 & 292 \\
\hline 9 & 91 & 36 & 144 & 8011 & 178 & 356 \\
\hline 11 & 133 & 44 & 176 & 17,293 & 262 & 524 \\
\hline 13 & 183 & 52 & 204 & 32,942 & 362 & 724 \\
\hline 16 & 273 & 64 & 256 & 73,712 & 542 & 1084 \\
\hline
\end{tabular}

in $H^{2}$ can be accomplished via generalized "row/column" routing, where a message is first routed in the "row" PDN and then in the "column" PDN, or vice versa. Similarly, broadcasting is done in two steps of row (column) broadcast followed by column (row) broadcast. Any sorting algorithm for a square mesh that uses row and column sorts as its basic components can be adapted to $H^{2}$ by emulating a linear-array sorting algorithm on the row and column PDNs. Hence, many algorithms developed for basic PDN lead directly to a number of corresponding algorithms for $H^{2}$ with little or no additional effort. Some examples are provided in a companion paper [27] that deals with the performance, algorithmic, and robustness attributes of PDNs.

\section{Topological Properties}

In addition to the network diameter, which is 2 in the case of basic PDNs, various other topological properties have a bearing on the performance of an interconnection network. In this section, we discuss two such properties: the average internode distance and the bisection width.

Theorem 3. The average internode distance of a PDN of order $\delta$ is $\Delta=2 \delta^{2} / n$.

Proof. Each node has distance of 0 to itself, 1 to its $2 \delta$ neighbors, and 2 to the other $\delta^{2}-\delta$ nodes. Hence, $\Delta=\left[2 \delta+2\left(\delta^{2}-\delta\right)\right] / n=2 \delta^{2} / n$. If we did not count the distance of a node to itself, the average internode distance would become $2 \delta^{2} /(n-1)=2 \delta /(\delta+1)$. However, the former result is somewhat more useful in that it makes it possible to find the average internode distance of a $q \mathrm{D}$ PDN by simply adding the $\Delta$ parameters of the component networks.

Theorem 4. For an element $s_{i}$ of a specific PDS of order $\delta$, define $s_{i}^{\prime}$ as $s_{i}$ if $s_{i}<n / 2$ and as $n-s_{i}$ if $s_{i}>n / 2$. The bisection width of a PDN based on this specific PDS is upper bounded by $\min \left(2 S_{\text {all }}^{\prime}, n M_{\text {odd }}-S_{\text {odd }}+S_{\text {even }}\right)$, where $M_{\text {odd }}$ is the number of odd elements in the PDS, $S_{\text {even }}$ and $S_{\text {odd }}$ represent the sum of all PDS elements that are even and odd, respectively, and $S_{\text {all }}^{\prime}$ is the sum of all $s_{i}^{\prime}$ values for the PDS.

Proof. An upper bound for bisection width is established by showing a specific bisection cut that requires removing that many links. Putting even and odd-numbered nodes on opposite sides and severing all the links between them is one way to find such an upper bound. Consider the links from node $i$ to nodes $i+s_{j}, 1 \leq j \leq \delta$ (connections from $i$ to $i-s_{j}$ will be counted from the other end, so we do not include them here). For $s_{j}$ even, the link goes from one side to the other side iff it wraps around and the odd number $n$ is subtracted from the true sum $i+s_{j}$, which has the same parity as $i$. For each even skip distance $s_{j}$, there are $s_{j}$ such links that go from one side to the other. For $s_{j}$ odd, on the other hand, the link from $i$ to $i+s_{j}$ goes to the other side only if there is no wraparound. For each odd skip distance $s_{j}$, there are $n-s_{j}$ such links. Thus, the total number of links going between odd and even nodes is

$$
\sum_{\text {odd skips }}\left(n-s_{j}\right)+\sum_{\text {even skips }} s_{j}=n M_{\text {odd }}-S_{\text {odd }}+S_{\text {even }} .
$$

Now, consider putting nodes 0 through $(n-1) / 2$ on one side and nodes $(n+1) / 2$ through $n-1$ on the other and severing all the links between the two sides. It is easy to see from a chordal-ring drawing of our PDN (as in Fig. 3) that $S_{\text {all }}^{\prime}$ links must be cut on each side of the ring to isolate the two parts. This is because $s_{j}$ nodes immediately before the cut have their $s_{j}$-type skip links cross to the other side for each $s_{j}<n / 2$ and $n-s_{i}$ nodes immediately after the cut have their $s_{j}$-type skip links cross to the other side for each $s_{j}>n / 2$.

Theorem 5. A lower bound on the bisection width of PDN is $\lceil(\delta+1)(n+1) / 4\rceil$. Together with the upper bound of Theorem 4, this implies that the bisection width of PDN is $\Theta\left(n^{1.5}\right)$, which is intermediate between the $\Theta(n)$ bisection of the hypercube and the $\Theta\left(n^{2}\right)$ bisection of $K_{n}$.

Proof. That the bisection width is $\mathrm{O}\left(n^{1.5}\right)$ is obvious by noting that each component of the upper bound derived in Theorem 4 is $\mathrm{O}\left(n^{1.5}\right)$. To complete the proof that the bisection width is $\Theta\left(n^{1.5}\right)$, we must demonstrate that the bisection width is $\Omega\left(n^{1.5}\right)$; that is, it must grow at least in proportion to $n^{1.5}$. One way of establishing a lower bound on the bisection width of a network is to demonstrate that $n(n-1) / 2$ routing paths between all pairs of nodes can be constructed such that $c$ or fewer of these paths pass through any given link [19]; we say that congestion of the constructed routing scheme is $c$. Then, in view of the fact that for odd $n,((n-1) / 2)((n+1) / 2)$ different routing paths among the $n(n-1) / 2$ constructed 
TABLE 3

Lower and Upper Bounds for the Bisection Width of Some PDNs Derived from Theorems 4 and 5 (Associated PDSs Appear in Table 1)

\begin{tabular}{|c|c|c|c|c|c|}
\hline $\boldsymbol{\delta}$ & $\boldsymbol{n}$ & $\Gamma(\boldsymbol{\delta}+\mathbf{1})(\boldsymbol{n}+\mathbf{1}) / \mathbf{4}\rceil$ & $\mathbf{2 S}_{\text {all }}^{\text {all }}$ & $\boldsymbol{n M}_{\text {odd }}-\boldsymbol{S}_{\text {odd }}+\boldsymbol{S}_{\text {even }}$ & Bisection range \\
\hline 2 & 7 & 6 & 8 & 10 & {$[6,8]$} \\
\hline 3 & 13 & 14 & 16 & 26 & {$[14,16]$} \\
\hline 4 & 21 & 28 & 34 & 54 & {$[28,34]$} \\
\hline 5 & 31 & 48 & 74 & 96 & {$[48,74]$} \\
\hline 7 & 57 & 116 & 164 & 288 & {$[116,164]$} \\
\hline 8 & 73 & 167 & 244 & 408 & {$[167,244]$} \\
\hline 9 & 91 & 230 & 342 & 476 & {$[230,342]$} \\
\hline 11 & 133 & 402 & 594 & 727 & {$[402,594]$} \\
\hline 13 & 183 & 644 & 836 & 1124 & {$[644,836]$} \\
\hline 16 & 273 & 1165 & 1844 & 2792 & {$[1165,1844]$} \\
\hline
\end{tabular}

above must cross each bisection of an $n$-node network, any bisection of our PDN must cut at least $\left(n^{2}-1\right) /(4 c)$ different links. To determine the value of $c$, we note that, in routing from a given node to all other nodes via shortest paths of length 1 or 2 , each backward link of the source node is used once, each forward link is used $\delta$ times, and each backward link of its neighbors is used at most once. This makes congestion on all links equal to $\delta$, given that each node is a forward neighbor of exactly $\delta$ other nodes and the backward link leading back to the intermediate node is never used. The conclusion that the bisection width is lower bounded by $\lceil(\delta+1)(n+1) / 4\rceil$ is immediate because

$\left(n^{2}-1\right) /(4 c)=(n-1)(n+1) /(4 \delta)=\left(\delta^{2}+\delta\right)(n+1) /(4 \delta)$.

Table 3 shows the bounds of Theorems 4 and 5 for the PDNs defined in Table 1. The exact bisection width of an arbitrary PDN is not yet known. Note that calculation of bisection width for an arbitrary graph is an NP-complete problem [12] and it remains so even for the class of regular graphs [8]. This explains the dearth of results on network bisection width.

\section{ConClusion}

We have introduced perfect difference networks and the mathematical underpinnings that make them desirable as robust, high-performance interconnection networks for parallel and distributed computation. Although other interconnection networks with topological and performance parameters similar to PDNs exist, we view these networks as worthy additions to the repertoire of computer system designers. Alternative network topologies offer additional design points that can be exploited to accommodate the needs of current and emerging technologies. Further study is needed to resolve some open questions and to derive cost/performance comparisons for PDNs and their derivatives. We offer some results along these lines in a companion paper that deals with routing problems, algorithm design issues, and robustness attributes of PDNs [27].

Basic PDNs have a diameter of 2 and a node degree of approximately $2 n^{1 / 2}$, which place them close to complete networks in terms of routing performance and much lower with respect to implementation cost. Not surprisingly, the exact average internode distance of an $n$-node PDN based on a PDS of order $\delta$ is $2-2(\delta+1) / n$; that is, very close to 2 . We have been unable to find the exact bisection width of a PDN but derived fairly tight bounds for it and, as a result, established that the bisection width is $\Theta\left(n^{1.5}\right)$.

Although PDNs are interesting and important as asymptotically optimal diameter-2 interconnection networks, it is much more likely that hybrid or composite networks involving PDNs as component structures will prove useful for practical applications. Here, we have introduced multidimensional PDNs as specific examples of such networks, but many other hybrid or composite structures are possible. For example, clustered PDNs based on swapped-network connectivity [44] merit attention. Such a swapped network consists of $n$ PDNs, each of which is of size $n$, with node $i$ in PDN $j$ connected to node $j$ in PDN $i$. The unused intercluster links of nodes $(i, i)$ can be assigned for input/output. This leads to a node degree of $2 \delta+1$. Network diameter is 5, compared to 4 for 2D PDNs, but this might be a worthwhile trade off given that node degree of the former is nearly half that of the latter. Like 2D PDNs, the node degree in these networks grows as the fourth root of the network size, leading to better scalability compared to basic or bipartite PDNs.

We previously noted that our focus in this paper is on simple difference sets that allow each value in $[1, n-1]$ to be derived as a difference $s_{i}-s_{j}$ in one, and only one, way. Clearly, nonsimple difference sets offer additional advantages with regard to fault tolerance, given multiple ways in which each value can be formed as a difference. However, these advantages come at the cost of a higher node degree. Possible generalizations of the perfect difference concept may lead to more efficient interconnection networks. For example, given our interest in 2-hop routing, we do not need to restrict ourselves to differences; sums can also be used. A natural question then is whether the use of difference/sum sets can lead to smaller sets (lower node degrees) or larger networks with the same node cost. Taking $n=15$ as an example, we note that the set $\{0,2,5,6\}$ leads to the mod-15 sums $\{2,5,6,7,8,11\}$ and mod-15 differences $\{13,10,9,2,12,11,5,3,14,6,4,1\}$, which together cover all integers in $[1,14]$. The set $\{0,2,5,6\}$ is not a perfect difference/sum set and, in fact, such a set might not exist. However, the concept may still warrant further 
investigation. Similarly, sets that yield each value as the sum of a small subset of size $k$ lead to networks with diameter $k$. Many other variations are also possible.

One direction for future research (suggested by one of the reviewers of this paper) is to pursue the use of perfect difference sets and other results from number theory in the design of networks that are closer to being strictly, rather than asymptotically, optimal. The node degree $2 \delta$ of PDNs is almost a factor of 2 above the optimal node degree suggested by Moore's bound. One may thus pursue the derivation of networks with roughly $\delta^{2}$ nodes whose node degree is $\delta+\varepsilon$ for a suitably small constant. It is the authors' belief that the introduction of PDNs based on perfect difference sets does not constitute the end of using number theory in deriving interesting interconnection networks but merely a beginning.

\section{REFERENCES}

[1] S.B. Akers and B. Krishnamurthy, "A Group-Theoretic Model for Symmetric Interconnection Networks," IEEE Trans. Computers, vol. 38, no. 4, pp. 555-566, Apr. 1989.

[2] A-E. Al-Ayyoub and K. Day, "The Hyperstar Interconnection Network," J. Parallel and Distributed Computing, vol. 48, no. 2, pp. 175-199, Feb. 1998.

[3] B.W. Arden and H. Lee, "Analysis of Chordal Ring Networks," IEEE Trans. Computers, vol. 30, no. 4, pp. 291-295, Apr. 1981.

[4] W.C. Arlinghaus, "Block Designs and Latin Squares," Applications of Discrete Math., J.G. Michaels and K.H. Rosen, eds., McGrawHill, 1991.

[5] A. Beutelspacher and U. Rosenbaum, Projective Geometry: From Foundations to Applications, section 2.8, Cambridge, pp. 81-88, 1998.

[6] L.N. Bhuyan and D.P. Agrawal, "Generalized Hypercube and Hyperbus Structures for a Computer Network," IEEE Trans. Computers, vol. 33, no. 4, pp. 323-333, Apr. 1984.

[7] N. Biggs, Algebraic Graph Theory. Cambridge Univ. Press, 1993.

[8] T.N. Bui, S. Chaudhuri, F.T. Leighton, and M. Sisper, "Graph Bisection Algorithms with Good Average Case Behavior," Combinatorica, vol. 7, no. 2, pp. 171-191, 1987.

[9] G.E. Carlson, J. Cruthirds, H. Section, and C. Wright, "Interconnection Networks Based on Generalization of Cube-Connected Cycles," IEEE Trans. Computers, vol. 34, pp. 769-772, Aug. 1985.

[10] W.J. Dally, "Performance Analysis of $k$-ary $n$-cube Interconnection Networks," IEEE Trans. Computers, vol. 39, no. 6, pp. 775-785, June 1990.

[11] T.A. Evans and H.B. Mann, "On Simple Difference Sets," Sankhya: Indian J. Statistics, vol. 11, pp. 357-364, 1951.

[12] M.R. Garey, D.D. Johnson, and L. Stockmeyer, "Some Simplified NP-Complete Graph Problems," Theoretical Computer Science, vol. 1, pp. 237-267, 1976.

[13] R.K. Guy, Unsolved Problems in Number Theory, second ed., Springer, pp. 118-121, 1994.

[14] M. Hall Jr., "A Survey of Difference Sets," Proc. Am. Math. Soc., vol. 7, pp. 975-986, 1956.

[15] M. Hall, Combinatorial Theory. Blaisdell, 1967.

[16] T.P. Kirkman, "On the Perfect r-Partitions of $r^{2}-r+1$," Trans. Historical Soc. of Lancashire and Cheshire, vol. 9, pp. 127-142, 1857.

[17] D.-M. Kwai and B. Parhami, "A Unified Formulation of Honeycomb and Diamond Networks," IEEE Trans. Parallel and Distributed Systems, vol. 12, no. 1, pp. 74-80, Jan. 2001.

[18] S. Lakshmivarahan, J.-S. Jwo, and S.K. Dahl, "Symmetry in Interconnection Networks Based on Cayley Graphs of Permutation Group: A Survey," Parallel Computing, vol. 19, pp. 361-401, 1993.

[19] F.T. Leighton, Introduction to Parallel Algorithms and Architectures: Arrays, Trees, and Hypercubes. Morgan Kaufmann, 1992.

[20] S. Lin and D.J. Costello Jr., Error Control Coding. Prentice-Hall, 1983.

[21] P.K. McKinley, Y.-j. Tsai, and D.F. Robinson, "Collective Communication in Wormhole-Routed Massively Parallel Computers," Computer, vol. 28, no. 12, pp. 39-50, Dec. 1995.

[22] L.M. Ni and P.K. McKinley, "A Survey of Wormhole Routing Techniques in Direct Networks," Computer, vol. 26, no. 2, pp. 6276, Feb. 1993.
[23] B. Parhami, Introduction to Parallel Processing: Algorithms and Architectures. Plenum Press, 1999.

[24] B. Parhami and D.-M. Kwai, "Periodically Regular Chordal Rings," IEEE Trans. Parallel and Distributed Systems, vol. 10, no. 6, pp. 658-672, June 1999.

[25] B. Parhami and D.-M. Kwai, "Challenges in Interconnection Network Design in the Era of Multiprocessor and Massively Parallel Microchips," Proc. Int'l Conf. Comm. in Computing, pp. 241246, June 2000.

[26] B. Parhami and D.-M. Kwai, "Incomplete $k$-ary $n$-cube and Its Derivatives," J. Parallel and Distributed Computing, vol. 64, no. 2, pp. 183-190, Feb. 2004.

[27] B. Parhami and M. Rakov, "Performance, Algorithmic, and Robustness Attributes of Perfect Difference Networks," IEEE. Trans. Parallel and Distributed Systems, vol. 16, no. 8, pp. 725-736, 2005.

[28] B. Parhami and C.-H. Yeh, "Why Network Diameter is Still Important," Proc. Int'l Conf. Comm. in Computing, pp. 271-274, June 2000.

[29] F.P. Preparata and J. Vuillemin, "The Cube-Connected Cycles: A Versatile Network for Parallel Computation," Comm. ACM, vol. 24, no. 5, pp. 300-309, May 1981.

[30] M. Rakov, "Method of Interconnecting Nodes and a Hyperstar Interconnection Structure," US Patent No. 5734 580, Mar. 1998.

[31] M. Rakov, "Hyperstar: A New Interconnection Topology," J. China Univ. of Posts and Telecomm., vol. 5, no. 2, pp. 10-18, Dec. 1998.

[32] M. Rakov, "Multidimensional Hyperstar and Hyperhub Interconnection Methods and Structures," US Patent Application No. 09/410 175, Sept. 1999.

[33] M. Rakov, "Hyperstar and Hyperhub Optical Networks Interconnection Methods and Structures," US Patent Application No. 09/634 129, Aug. 2000.

[34] M. Rakov and J. Mackall, "Method of Interconnecting Functional Nodes and a Hyperstar Interconnection Structure," US Patent No. 6330 706, Dec. 2001.

[35] M. Rakov and O.A. Vakulskiy, "Computer Systems Design Using Apparatus of the Ideal Ring Proportions," Theses of the Int'l Conf. New Information Technologies, Voronezh Polytechnic Inst., (in Russian), pp. 77-78, 1992.

[36] M. Rakov, O. Vakulskiy, and I. Stetsko, "Using the Ideal Code Proportion Apparatus for Improving the Local Nets Main Characteristics," Proc. Int'l Seminar on Local Area Networks, Riga, IEVT, (in Russian), pp. 31-36, 1992.

[37] J.P. Robinson and A.J. Bernstein, "A Class of Binary Recurrent Codes with Limited Error Propagation," IEEE Trans. Information Theory, vol. 13, no. 1, pp. 106-113, Jan. 1967.

[38] J. Singer, "A Theorem in Finite Projective Geometry and Some Applications to Number Theory," Trans. Am. Math. Soc., vol. 43, pp. 377-385, 1938.

[39] M.B. Sverdlik and A.N. Meleshkevich, "Synthesis of Optimum Pulsed Sequences Having the Property of 'No More than One Coincidence,'" Radio Eng. and Electronic Physics, vol. 19, no. 4, pp. 46-54, 1974.

[40] M. Sverdlik, Optimal Discrete Signals, Moscow, Soviet Radio, (in Russian), 1975.

[41] M.B. Sverdlik and A.N. Meleshkevich, "Table of Optimal Sets with the Property of 'No More than One Coincidence,'" Radio Eng. and Electronic Physics, vol. 20, no. 6, pp. 148-150, 1975.

[42] M.B. Sverdlik and A.N. Meleshkevich, "Synthesis of Ensembles of Pulse Sequences with Properties of 'No More than One Coincidence,'" Radio Eng. and Electronic Physics, vol. 21, no. 7, pp. 61-68, 1976.

[43] J.H. van Lint and R.M. Wilson, A Course in Combinatorics, (see chapter 27 entitled "Difference Sets and Automorphisms"), Cambridge Univ. Press, 1992.

[44] C.-H. Yeh and B. Parhami, "Swapped Networks: Unifying the Architectures and Algorithms of a Wide Class of Hierarchical Parallel Processors," Proc. Int'l Conf. Parallel and Distributed Systems, pp. 230-237, June 1996.

[45] A. Youssef, "Design and Analysis of Product Networks," Proc. Symp. Frontiers of Massively Parallel Computation, pp. 521-528, Feb. 1995.

[46] S.G. Ziavras, Q. Wang, and P. Papathanasiou, "Viable Architectures for High-Performance Computing," The Computer J., vol. 46, no. 1, pp. 36-54, 2003. 


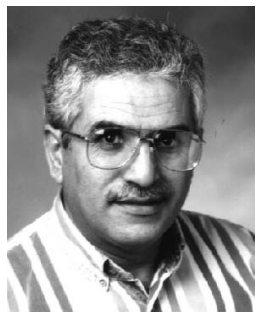

Behrooz Parhami received the $\mathrm{PhD}$ degree in computer science from the University of California, Los Angeles, in 1973. Presently, he is a professor in the Department of Electrical and Computer Engineering, University of California, Santa Barbara. His research deals with parallel architectures and algorithms, computer arithmetic, and reliable computing. In his previous position with Sharif University of Technology in Tehran, Iran (1974-1988), he was also involved in the areas of educational planning, curriculum development, standardization efforts, technology transfer, and various editorial responsibilities, including a five-year term as editor of Computer Report, a Farsi-language computing periodical. Dr. Parhami's technical publications include more than 220 papers in journals and international conferences, a Farsilanguage textbook, and an English/Farsi glossary of computing terms. Among his latest publications are two graduate-level textbooks on parallel processing (Plenum, 1999) and computer arithmetic (Oxford, 2000) and an introductory textbook on computer architecture (Oxford, 2005). Dr. Parhami is a fellow of the IEEE and the IEEE Computer Society, a chartered fellow of the British Computer Society, a member of the Association for Computing Machinery, and a distinguished member of the Informatics Society of Iran, for which he served as a founding member and President from 1979-1984. He also served as chairman of IEEE Iran Section (1977-1986) and received the IEEE Centennial Medal in 1984.

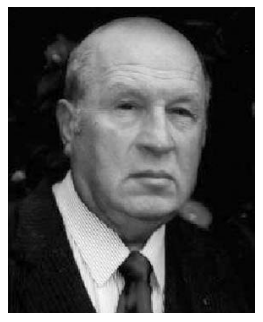

Mikhail A. Rakov graduated (summa cum laude) from L'vov Polytechnic Institute in 1956 with a BSci degree in electrical engineering. He received the PhD degree from the Kiev Institute of Electrotechnics in 1962 and the DSci degree from the Moscow Institute of Energetics in 1971. From 1959 to 1993, he held positions of increasing responsibility at the Institute of Physics and Mechanics, Ukrainian Academy of Sciences, where he conducted research on multiple-valued logic, abstract algebraic systems, digital signal processing, and network architecture. The main focus of this research was on developing practical applications for advanced mathematical constructs. He was appointed a professor of information theory and computer science in 1978 and was elected a member of the International Academy of Informatization in 1993. Dr. Rakov is author or coauthor of 10 scientific books, more than 250 published articles, and more than 100 patents in the former Soviet Union and in the US. Since emigrating to the US, he has been working as an independent scientific consultant. He has been affiliated with the Computer Science Department at the University of California, Santa Barbara, as a professional researcher since 2002.

$\triangleright$ For more information on this or any other computing topic, please visit our Digital Library at www.computer.org/publications/dlib. 